\title{
Neural correlates of enhanced executive functions : is less more?
}

\section{Putkinen, Vesa}

2018-07

Putkinen , V \& Saarikivi , K 2018 , ' Neural correlates of enhanced executive functions : is less more? ' , Annals of the New York Academy of Sciences , vol. 1423 , no. 1 , pp. 117-125 . https://doi.org/10.1111/nyas.13645

http://hdl.handle.net/10138/304270

https://doi.org/10.1111/nyas.13645

unspecified

publishedVersion

Downloaded from Helda, University of Helsinki institutional repository.

This is an electronic reprint of the original article.

This reprint may differ from the original in pagination and typographic detail.

Please cite the original version. 


\title{
Neural correlates of enhanced executive functions: is less more?
}

\author{
Vesa Putkinen ${ }^{1,2}$ and Katri Saarikivi ${ }^{2}$ \\ ${ }^{1}$ Turku PET Centre, University of Turku, Turku, Finland ${ }^{2}$ Cognitive Brain Research Unit, Department of Psychology and \\ Logopedics, Faculty of Medicine, University of Helsinki, Helsinki, Finland \\ Address for correspondence: Vesa Putkinen, Turku PET Centre, University of Turku, Turku, FI-20521, Finland. \\ vesa.putkinen@utu.fi
}

\begin{abstract}
Musical training has been associated with superior performance in various executive function tasks. To date, only a few neuroimaging studies have investigated the neural substrates of the supposed "musician advantage" in executive functions, precluding definite conclusions about its neural basis. Here, we provide a selective review of neuroimaging studies on plasticity and typical maturation of executive functions, with the aim of investigating how proficient performance in executive function tasks is reflected in brain activity. Specifically, we examine the evidence for the hypothesis that enhanced or mature executive functions are manifested as efficient use of neural systems supporting those functions. We also present preliminary results from a functional magnetic resonance imaging study suggestingin line with this hypothesis-that musically trained adolescents recruit frontoparietal regions less strongly during executive functions tasks than untrained peers.
\end{abstract}

Keywords: musical training; executive functions; fMRI; adolescence; neural efficacy

\section{Introduction}

"How can less be more? That's impossible. More is more." -Yngwie J. Malmsteen

Interindividual variation in cognitive skills has been among the most intensely studied subjects in experimental psychology since the advent of the discipline, and, recently, understanding the brain basis of such individual differences has become one of the central goals of cognitive neuroscience. ${ }^{1}$ One trend that is emerging from this line of research is that higher performance in trait and task measures of cognitive ability tends to be associated with lower blood oxygen-level dependent signal in the related neural structures. Such findings are commonly presumed to reflect efficient use of neural resources in high-performing individuals due to lower need for effort or reduced cognitive load and, conversely, recruitment of compensatory or inessential neural resources in low-performing individuals. This is idea is akin to the neural efficiency hypothesis originating from studies that have examined the neural basis of intelligence ${ }^{2}$ (for a critical discussion, see
Ref. 3) and has more recently found support in studies examining the neural bases of executive functions. Here, our goal is to examine how these results might inform studies on differences in executive functions between musicians and nonmusicians.

Executive functions encompass top-down control mechanisms that support higher order processes, such as planning, decision making, and self-control. ${ }^{4}$ Studies in children and adults indicate that performance in different executive function tasks tends to cluster in a way that suggests partially distinct but correlated subcomponents of executive functions. ${ }^{5-7}$ On the basis of such findings, an influential model of executive functions posits inhibition, set shifting, and working memory as the core components of executive functions. ${ }^{8}$ Inhibition refers to the overriding of reflexive or habitual behavior or cognitive processes, while setshifting involves switching between response strategies or mental states. Finally, working memory refers to a limited-capacity system for temporary storage and manipulation of recently presented or retrieved information. 
In adults, executive functions rely heavily on the frontal lobe but also recruit parietal regions as well as subcortical structures, such as the thalamus, basal ganglia, and the cerebellum. ${ }^{9-11}$ These regions have been proposed to support a range of executive functions and thereby to form a domain-general cognitive control network. Accordingly, working memory, inhibition, and set-shifting tasks have all been consistently found to activate these regions, albeit with different weighting across the network. ${ }^{11}$

Performance in executive function tasks varies substantially among individuals, and these individual differences predict various societally important phenomena ranging from academic performance to healthy lifestyle choices, while early deficits in executive functions contribute to learning difficulties. ${ }^{12}$ Consequently, there is considerable academic interest in the neural underpinnings of executive functions, their maturation, and factors that support their development. Executive functions appear early in development, mature rapidly during preschool age, ${ }^{13}$ and continue to develop in school age and adolescence, coinciding with developmental changes in the structure and function of the prefrontal cortex. ${ }^{14,15}$ Various training programs have been found to support the development of executive functions, indicating that they are malleable by experience in childhood (for a review, see Ref. 16).

Interestingly, musically trained individuals have been found to outperform untrained ones in different executive function tasks. ${ }^{17}$ These include inhibition tasks, such as the Simon, ${ }^{18}$ Stroop, ${ }^{18}$ and go-no go ${ }^{19}$ tasks; working memory tasks, such as the backward letter, ${ }^{20}$ digit span, ${ }^{21,22}$ and $n$-back ${ }^{23}$ tasks; and set-shifting tasks, such as trail-making task ${ }^{22}$ and other switching/dual tasks. ${ }^{24}$ Of the three components of executive functions that are our focus here, arguably most consistent evidence exists for a musician advantage in working memory. ${ }^{25}$ Collectively, these findings suggest that the demands of learning to play a musical instrument might train domaingeneral executive functions or, alternatively, that efficient executive functions help one to persist in musical training.

For the past 14 years, a longitudinal study conducted at the University of Helsinki has investigated different aspects of brain maturation in musically trained and untrained children and adolescents. ${ }^{26-28}$ The majority of the subjects have participated in the study from age 7 onward and were 14-21 years old at the time of writing. The participants in the music group started playing an instrument around the age of 7 when they entered a public elementary school that emphasizes music in the curriculum. The untrained participants have no formal musical training, have attended standard elementary school, and are matched in age and socioeconomic status (parental income and education) to the music group. The first measurements in the longitudinal study focused on auditory event-related potentials (ERPs), the mismatch negativity in particular, while the more recent measurements also included behavioral, ERP, and functional magnetic resonance imaging (fMRI) measures of executive functions. ${ }^{28,29} \mathrm{In}$ one of our studies, ${ }^{29}$ we administered the inhibition and set-shifting subtests from a standardized test battery of executive functions ${ }^{30}$ to 90 of the children and adolescents in our sample, then aged 9-15 years. We found that the musically trained children outperformed the untrained ones in both tasks irrespective of age. In contrast, we found no evidence for a group difference in other neurocognitive tests, including measures of general cognitive ability (as indexed by the vocabulary and block-design subtests from the WISC-IV battery). This suggests that the advantage in the inhibition and set-shifting tasks did not simply reflect generally higher cognitive performance in the musically trained participants.

It is a nontrivial challenge to predict how such advantages in executive functions are reflected in brain function. Namely, high task performance has been sometimes associated with stronger or differentially distributed activity within the same network as low task performance or the activation of different regions than less proficient performance (for a discussion on neuroimaging of individual differences in cognitive performance, see Ref. 31). For studies in children, this issue is even more complex, as the manner in which proficient executive functions are reflected in brain activity might change during the course of brain maturation. ${ }^{32}$ However, there is ample evidence that proficient cognitive performance is under many circumstances reflected in decreased activation in the same structures as less proficient performance. Below, we examine the rationale for the hypothesis that such neural efficiency might underlie the superior performance reported in musically trained individuals executive function tasks by giving a selective review of (1) neuroimaging studies that have investigated the 
effects of cognitive training on brain functions, (2) studies in bilinguals who tend to show enhanced executive functions, and (3) studies on neurodevelopmental changes underlying the maturation of executive functions. Finally, we present preliminary results from an fMRI study in line with this hypothesis and contrast these results with the existing neuroimaging literature on executive functions in adult musicians and musically trained children.

\section{Neural effects of cognitive training}

Studies on the efficacy of executive function training regimes have demonstrated that performance in specific tasks tapping into these functions can be improved by training in typically developing children, healthy adults, and different patient groups. ${ }^{16}$ The effects of working memory training, often with adaptive $n$-back tasks, ${ }^{33}$ have received particularly close attention, but training-related improvements in other facets of executive functions have also been reported. ${ }^{34,35}$ Whether these training benefits generalize to nontrained tasks or can have positive impact on real-life outcomes, such as academic performance, remains debated. ${ }^{33,36}$ In any case, assuming that musical training indeed benefits executive functions, studies examining brain activity before and after executive function training may offer insight into the kinds of functional differences that could be expected between musicians and nonmusicians during executive function tasks.

Numerous neuroimaging studies have taken this approach in healthy adults, and the majority of them have found evidence for training-induced decreases in activity in frontal regions. ${ }^{37-44}$ For instance, a recent fMRI study found that participants who were randomly assigned to inhibition training improved significantly more in a task requiring the stopping of an ongoing motor response (stop signal task) than a control group who had received sham training and, further, that the magnitude of behavioral improvement correlated with decreases in dorsolateral prefrontal cortex (DLPFC) activity during inhibition trials ${ }^{45}$ (see also, Ref. 38). As an example from working memory training, one study examined how training in a visual or auditory $n$-back task altered brain activity during a visual $n$-back task and found that both training regimes lead to a posttraining activation decrease in frontal and parietal regions when compared to a control group without training. ${ }^{43}$ Imaging studies have also examined the effects of training aiming to counteract agingrelated declines in working memory performance and found posttraining decrease in brain activity in frontal regions. ${ }^{46,47}$ To our knowledge, no published study has randomly assigned healthy children to executive function training and a control condition and measured task-related brain activity fMRI pre- and posttraining. From other domains of training in adolescents, an early study found that training in algebra led to reduced activity in frontoparietal regions during equation solving, ${ }^{48}$ while a subsequent study found reduced prefrontal activity after training in playing Tetris. ${ }^{49}$

There seems to be relatively consistent evidence for executive function training-induced reductions in recruitment of frontal and parietal regions in adults and some evidence suggesting that the same is true in adolescents, while almost nothing is known about the effects of cognitive training on brain activity in healthy children.

\section{Neural correlates of executive functions in bilinguals}

In addition to the effects of musical training, the influence of bilingualism on brain function and structure is another popular model for plastic changes in the neural mechanisms supporting executive functions induced by long-term experience. The rationale is that the need to manage two languages trains executive functions, as it necessitates flexible switching between the languages and the selection of the contextually appropriate one while inhibiting the other. Indeed, there is considerable evidence that bilinguals show enhanced executive functions ${ }^{50}$ (however, see Ref. 51). Neuroimaging studies comparing bilinguals and monolinguals broadly support the notion that bilinguals show attenuated activity in frontal regions during executive function tasks.

One of these studies found that bilinguals engaged the anterior cingulate (ACC) less than monolinguals when performing a Flanker task, suggesting more efficient inhibition and conflict processing in bilinguals when compared with monolinguals. ${ }^{52}$ This finding was replicated in a more recent fMRI study ${ }^{53}$ that found that bilinguals showed less activation in ACC during a stop-signal inhibition task. Finally, a study in younger and older bi- and monolingual individuals used a task requiring the switching between categorizing visual stimuli by their 
color versus shape and found that the bilinguals outperformed the monolinguals and, further, that older monolinguals engaged frontal regions more strongly than younger monolinguals, whereas similar differences were not observed in the bilingual subjects. ${ }^{54}$ These results suggest that bilingualism can counteract age-related decline in executive functions and the associated reduction in efficiency of the underlying neural mechanisms.

\section{Maturation of the neural circuits supporting executive functions in adolescence}

One proposed mechanism for training-induced improvements in children's executive functions is that the training influences the construction of the neural networks supporting task performance and in essence accelerates the maturation of these networks. ${ }^{55}$ The anatomy of the cerebral cortex shows regionally heterogeneous developmental trajectories that are particularly protracted in higher order association areas supporting executive functions. ${ }^{56-59}$ Functional imaging studies indicate that the structural maturation of these circuits is paralleled by a complex pattern of age-related increases, decreases, and redistribution in activation in neural networks supporting executive functions. ${ }^{32}$ One fairly consistent finding, however, is that children and adolescents recruit prefrontal regions more strongly during executive function tasks than adults, suggesting that young subjects need to engage additional neural resources in order to perform such tasks successfully.

Early small-scale fMRI studies employing go-no go tasks reported initial evidence for an increasing efficiency with age in the neural substrates of executive functions. Namely, one study found more focal activation in frontal cortical regions in in adults (20-24 years, $n=9)$ than in children (712 years, $n=9),{ }^{60}$ while another study found a negative correlation with age ( $8-20$ years, $N=19)$ and the recruitment of the left middle/superior frontal gyri during the task. ${ }^{61}$ A subsequent study with a substantially larger sample size $(N=98)$ found that children (aged 8-12 years) recruited the DLPFC more strongly than adolescents (13-17 years) or adults (18-27 years) in an antisaccade task that requires the participant to override the prepotent saccade to a peripheral stimulus and make a voluntary saccade to the opposite direction. ${ }^{62-64}$ A recent large-scale $(N=123)$ longitudinal study corroborated this finding. ${ }^{65}$ Namely, the study provided compelling evidence for an age-related reduction in the engagement of the right DLPFC in the antisaccade task throughout childhood and adolescence. Along the same lines, another recent longitudinal fMRI study found age-related activity decreases during working memory maintenance in frontal regions between childhood and mid-adolescence. ${ }^{66}$ In keeping with these studies, another study found that adolescents engaging in frequent heavy drinking showed increased prefrontal activity during a go-no go inhibition task relative to nondrinking peers. ${ }^{67}$ A study in younger children (5-10 years) found age-related reductions in medial prefrontal cortex activity during trials of an inhibition task (Simon task) that required a motor response with the hand that was incompatible with the spatial location of a visual stimulus (i.e., right hand for stimuli appearing on the left and vice versa). Although the complex neurodevelopmental patterns underlying the maturation of executive functions clearly involve more than mere reduction of prefrontal activity, ${ }^{32}$ together these studies suggest an agerelated reduction in the reliance on prefrontal structures in various executive function tasks.

\section{Preliminary behavioral and fMRI results from the Helsinki longitudinal study}

Recently, a subset of the subjects ( $n=49,26$ musically trained, 30 females) in our longitudinal study, aged 16-21 years at the time, participated in a crosssectional fMRI experiment investigating the neural underpinnings of the enhanced inhibition and setshifting in the music group observed in our previous study. ${ }^{29}$

The experiment consists of four naming, four inhibition, and eight set-shifting task blocks based on the Nespy test employed in our previous study, ${ }^{29}$ presented in two runs in a random order. On a given trial, the participant is shown a white or black arrow at the center of the screen pointing to the left or right and is asked to respond with a button press according to the instructions shown on the screen before each block. In the baseline blocks, the task was simply to press the response button with the hand corresponding to the direction of the arrow (i.e., press a button with the right hand if the arrow points to the right and vice versa). In the inhibition blocks, the task was to suppress the tendency to 
respond with the congruent hand and instead respond with the opposite hand to the direction of the arrow (i.e., press a button with the right hand if the arrow points left and vice versa). In baseline and inhibition conditions, the color of the arrow was task irrelevant. In the set-shifting blocks, in contrast, the participant was instructed to respond with the congruent or incongruent hand depending on the color of the arrow. In half of the set-shifting blocks, black arrows require the incongruent response, and white arrows require the congruent response. In the other half of these blocks, the color-response rule mapping is reversed. Thus, the set-shifting task requires switching between the congruent trials that correspond to the baseline condition and the incongruent trials that correspond to the inhibition condition. Our main interest was to investigate how the additional shifting and working memory demands in the set-shifting condition would affect the contrast between the shifting-congruent versus baseline and shifting-incongruent versus inhibition contrasts and how brain activity revealed by these contrasts differ between the groups. These comparisons are made between conditions that are identical in terms of stimuli and stimulusresponse mapping but differ in cognitive demands.

Only the contrast between the incongruent trials in the set-shifting condition and the inhibition trials revealed a group difference. For this contrast, the control group showed stronger activity than the music group in the right inferior frontal cortex and the right intraparietal sulcus (Fig. 1). The opposite contrast (music $>$ control) did not reveal signifi- cant group differences. Thus, the results suggest the music group was able to perform the task with more efficient usage of neural resources than the control group.

It is noteworthy that the effect was observed for a nonmusical visual task, suggesting a domaingeneral enhancement of the neural systems supporting executive functions in the musically trained adolescents and young adults. Like many complex executive function tasks, the incongruent trials of the set-shifting condition tapped into multiple subcomponents of executive functions. In addition to set shifting per se, these trials required inhibition of the congruent response, while the condition as a whole required maintenance of two responsestimulus mappings in working memory. The finding that these trials recruited right prefrontal and parietal regions in the control group dovetails with previous imaging studies that have consistently found activity in these regions in inhibition tasks that require working memory updating. ${ }^{66}$

\section{Previous studies on musical training and the neural correlates of executive functions}

Only a handful of fMRI studies have examined the neural underpinnings of executive functions in musicians and nonmusicians. The first of these studies concentrated on working memory and used musical stimuli such as melodies ${ }^{68,69}$ or chords. ${ }^{70}$ Here, we focus on studies that, like ours, have used nonmusical tasks and thereby minimize the

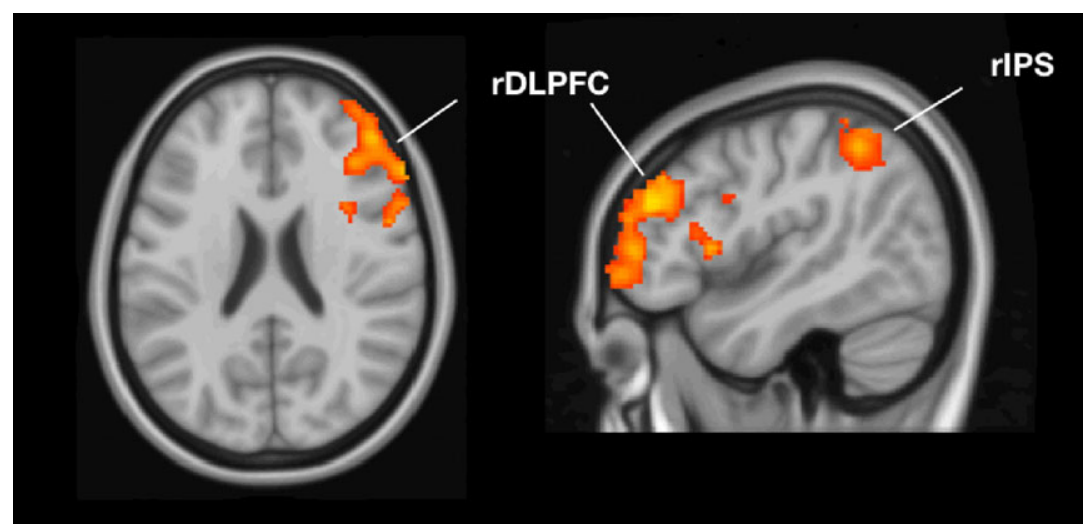

Figure 1. The control groups showed higher activity $(P<0.05$, corrected for multiple comparisons at the cluster level $)$ than the music group for the set-shifting incongruent inhibition contrast in the DLPFC (MNI peak coordinates $x=46, y=42, z=26$ ) and IPS $(x=38, y=-44, z=48)$. 
contribution of stimulus familiarity and explicit musical knowledge.

A recent study by Alain et al. ${ }^{71}$ found reduced activation in the DLPFC and superior frontal gyrus in adult musicians compared with nonmusicians in an auditory $n$-back working memory task with environmental sounds as stimuli. These results suggest lower recruitment of neural resources in adult musicians. In children, one study found that 9to 12-year-old musically trained children recruited the supplemental motor area and ventrolateral prefrontal cortex more strongly than untrained children in an audiovisual set-shifting task. ${ }^{22}$ Along the same lines, a subsequent study focused on inhibition found that school-aged children who had participated in a music training program for 2 years showed stronger activation in the ACC, inferior frontal gyrus, pre-supplementary motor area (SMA)/SMA, and insula on the incongruent trials of a color-word Stroop task relative to an active control group participating in a sports training and a passive control group who did not participate in an extracurricular program. ${ }^{72}$

It is not possible to draw definite conclusions about the neural basis of the supposed musician advantage in executive functions based on such a scarce literature, especially since these studies have targeted different (albeit related) executive functions. Nonetheless, these studies tentatively suggest that adult musicians show decreased activity in more anterior prefrontal regions, while musically trained children display increased activity in more posterior frontal cortex (as indicated by the peak voxel coordinates) and other structures during executive function tasks. The latter findings could be interpreted as evidence that musically trained children are able to engage neural resources that are inaccessible to untrained children, perhaps because of earlier maturation, stronger task engagement, or the use of different cognitive strategies. It is possible that several mechanisms underlie the putative musician advantage in executive functions, and it seems plausible that their relative contribution might depend on the maturational state of the brain.

\section{Conclusions}

The assertion that expert performance requires fewer neural resources than that of novices has relatively strong empirical support. ${ }^{2}$ Here, we reviewed evidence in favor of this neural efficiency hypothesis in the domain of executive functions from studies that have linked reduction in frontal and parietal activity with experience-induced enhancement of inhibition, set shifting, and working memory, as well as the maturation of these functions. Our preliminary fMRI results from musically trained adolescents and young adults are consistent with the notion that musical expertise is also associated with the efficiency of a right-lateralized frontoparietal network activated by the executive function tasks in which the music group outperformed the control group in our previous behavioral study. ${ }^{29}$

The question remains as to what degree the superior executive functions in musicians are a beneficial side effect of musical training or whether they stem from other factors (for recent critical reviews on far transfer effects, see Refs. 73 and 74). Twin studies have provided evidence for genetic etiology of individual differences in executive functions, ${ }^{75}$ and, while heritability and plasticity are not mutually exclusive, such findings suggest that the enhanced executive functions in musicians might be partly attributable to genetic predispositions rather than experience. Indeed, it is widely accepted that both genetic and training-related factors likely contribute to the cognitive benefits associated with musical training. ${ }^{76}$ Since all of the published imaging studies on the neural substrates of executive functions in musicians and nonmusicians are cross-sectional, there is a need for longitudinal studies with pretraining measures assessing the dynamics of brain activity within the same musically trained and untrained subjects during the course of training. Finally, although we have concentrated on univariate analyses of fMRI data, network-level analyses, as well as other brain research methods, such as electroencephalography and magnetoencephalography, can provide important insight into experience-dependent plasticity and predispositions in brain function that underlie the supposed musician advantage in executive functions.

\section{Competing interests}

The authors declare no competing interests.

\section{References}

1. Dubois, J. \& R. Adolphs. 2016. Building a science of individual differences from fMRI. Trends Cogn. Sci. 20: 425-443.

2. Neubauer, A.C. \& A. Fink. 2009. Intelligence and neural efficiency. Neurosci. Biobehav. Rev. 33: 1004-1023. 
3. Poldrack, R.A. 2015. Is "efficiency" a useful concept in cognitive neuroscience? Dev. Cogn. Neurosci. 11: 12-17.

4. Diamond, A. 2013. Executive functions. Annu. Rev. Psychol. 64: $135-168$.

5. Huizinga, M., C.V. Dolan \& M.W. van der Molen. 2006. Agerelated change in executive function: developmental trends and a latent variable analysis. Neuropsychologia 44: 20172036.

6. Miyake, A., N.P. Friedman, M.J. Emerson, et al. 2000. The unity and diversity of executive functions and their contributions to complex "frontal lobe" tasks: a latent variable analysis. Cogn. Psychol. 41: 49-100.

7. Miyake, A. \& N.P. Friedman. 2012. The nature and organization of individual differences in executive functions four general conclusions. Curr. Dir. Psychol. Sci. 21: 8-14.

8. Friedman, N.P. \& A. Miyake. 2017. Unity and diversity of executive functions: individual differences as a window on cognitive structure. Cortex 86: 186-204.

9. Duncan, J. 2010. The multiple-demand (MD) system of the primate brain: mental programs for intelligent behaviour. Trends Cogn. Sci. 14: 172-179.

10. Nee, D.E., T.D. Wager \& J. Jonides. 2007. Interference resolution: insights from a meta-analysis of neuroimaging tasks. Cogn. Affect. Behav. Neurosci. 7: 1-17.

11. Niendam, T.A., A.R. Laird, K.L. Ray, et al. 2012. Metaanalytic evidence for a superordinate cognitive control network subserving diverse executive functions. Cogn. Affect. Behav. Neurosci. 12: 241-268.

12. Titz, C. \& J. Karbach. 2014. Working memory and executive functions: effects of training on academic achievement. Psychol. Res. 78: 852-868.

13. Garon, N., S.E. Bryson \& I.M. Smith. 2008. Executive function in preschoolers: a review using an integrative framework. Psychol. Bull. 134: 31-60.

14. Best, J.R. \& P.H. Miller. 2010. A developmental perspective on executive function. Child Dev. 81: 1641-1660.

15. Blakemore, S.-J. \& S. Choudhury. 2006. Development of the adolescent brain: implications for executive function and social cognition. J. Child Psychol. Psychiatry 47: 296-312.

16. Diamond, A. \& D.S. Ling. 2016. Conclusions about interventions, programs, and approaches for improving executive functions that appear justified and those that, despite much hype, do not. Dev. Cogn. Neurosci. 18: 34-48.

17. Moreno, S. \& G.M. Bidelman. 2014. Examining neural plasticity and cognitive benefit through the unique lens of musical training. Hear. Res. 308: 84-97.

18. Bialystok, E. \& A.-M. DePape. 2009. Musical expertise, bilingualism, and executive functioning. J. Exp. Psychol. Hum. Percept. Perform. 53: 565-574.

19. Moreno, S., E. Bialystok, R. Barac, et al. 2011. Short-term music training enhances verbal intelligence and executive function. Psychol. Sci. 22: 1425-1433.

20. George, E.M. \& D. Coch. 2011. Music training and working memory: an ERP study. Neuropsychologia 49: 10831094.

21. Clayton, K.K., J. Swaminathan, A. Yazdanbakhsh, et al. 2016. Executive function, visual attention and the cocktail party problem in musicians and non-musicians. PLoS One 11: e0157638.
22. Zuk, J., C. Benjamin, A. Kenyon, et al. 2014. Behavioral and neural correlates of executive functioning in musicians and non-musicians. PLoS One 9: e99868.

23. Slevc, L.R., N.S. Davey, M. Buschkuehl, et al. 2016. Tuning the mind: exploring the connections between musical ability and executive functions. Cognition 152: 199-211.

24. Moradzadeh, L., G. Blumenthal \& M. Wiseheart. 2015. Musical training, bilingualism, and executive function: a closer look at task switching and dual-task performance. Cogn. Sci. 39: 992-1020.

25. Talamini, F., G. Altoè, B. Carretti, et al. 2017. Musicians have better memory than nonmusicians: a meta-analysis. PLoS One 12: e0186773.

26. Putkinen V., M. Tervaniemi, K. Saarikivi, et al. 2014. Investigating the effects of musical training on functional brain development with a novel melodic MMN paradigm. Neurobiol. Learn. Mem. 110: 8-15.

27. Putkinen, V., M. Tervaniemi, K. Saarikivi, et al. 2014. Enhanced development of auditory change detection in musically trained school-aged children: a longitudinal eventrelated potential study. Dev. Sci. 17: 282-297.

28. Putkinen, V., M. Tervaniemi, K. Saarikivi, et al. 2015. Promises of formal and informal musical activities in advancing neurocognitive development throughout childhood: musical activities in neurocognitive development. Ann. N.Y. Acad. Sci. 1337: 153-162.

29. Saarikivi, K., V. Putkinen, M. Tervaniemi, et al. 2016. Cognitive flexibility modulates maturation and music-trainingrelated changes in neural sound discrimination. Eur. J. Neurosci. 44: 1815-1825.

30. Korkman, M. 2008. Nepsy II. Helsinki: Psykologien Kustannus Oy.

31. Yarkoni, T. \& T.S. Braver. 2010. Cognitive neuroscience approaches to individual differences in working memory and executive control: conceptual and methodological issues. In Handbook of Individual Differences in Cognition. 87-107. New York: Springer.

32. Luna, B., A. Padmanabhan \& K. O’Hearn. 2010. What has fMRI told us about the development of cognitive control through adolescence? Brain Cogn. 72: 101-113.

33. Au, J., E. Sheehan, N. Tsai, et al. 2015. Improving fluid intelligence with training on working memory: a meta-analysis. Psychon. Bull. Rev. 22: 366-377.

34. Kueider, A.M., J.M. Parisi, A.L. Gross, et al. 2012. Computerized cognitive training with older adults: a systematic review. PLoS One 7: e40588.

35. Spierer, L., C.F. Chavan \& A.L. Manuel. 2013. Traininginduced behavioral and brain plasticity in inhibitory control. Front. Hum. Neurosci. 7: 427.

36. Melby-Lervåg, M. \& C. Hulme. 2016. There is no convincing evidence that working memory training is effective: a reply to $\mathrm{Au}$ et al. (2014) and Karbach and Verhaeghen (2014). Psychon. Bull. Rev. 23: 324-330.

37. Beauchamp, K.G., L.E. Kahn \& E.T. Berkman. 2016. Does inhibitory control training transfer?: behavioral and neural effects on an untrained emotion regulation task. Soc. Cogn. Affect. Neurosci. 11: 1374-1382.

38. Chavan, C.F., M. Mouthon, B. Draganski, et al. 2015. Differential patterns of functional and structural plasticity within 
and between inferior frontal gyri support training-induced improvements in inhibitory control proficiency. Hum. Brain Mapp. 36: 2527-2543.

39. Hempel, A., F.L. Giesel, N.M. Garcia Caraballo, et al. 2004. Plasticity of cortical activation related to working memory during training. Am. J. Psychiatry 161: 745-747.

40. Jansma, J.M., N.F. Ramsey, H.A. Slagter, et al. 2001. Functional anatomical correlates of controlled and automatic processing. J. Cogn. Neurosci. 13: 730-743.

41. Landau, S.M., E.H. Schumacher, H. Garavan, et al. 2004. A functional MRI study of the influence of practice on component processes of working memory. Neuroimage 22: 211221.

42. Sayala, S., J.B. Sala \& S.M. Courtney. 2005. Increased neural efficiency with repeated performance of a working memory task is information-type dependent. Cereb. Cortex 16: 609617.

43. Schneiders, J.A., B. Opitz, C.M. Krick, et al. 2011. Separating intra-modal and across-modal training effects in visual working memory: an fMRI investigation. Cereb. Cortex 21: 2555-2564.

44. Tomasi, D., T. Ernst, E.C. Caparelli, et al. 2004. Practiceinduced changes of brain function during visual attention: a parametric fMRI study at 4 Tesla. Neuroimage 23: 14141421.

45. Berkman, E.T., L.E. Kahn \& J.S. Merchant. 2014. Traininginduced changes in inhibitory control network activity. J. Neurosci. 34: 149-157.

46. Brehmer, Y., A. Rieckmann, M. Bellander, et al. 2011. Neural correlates of training-related working-memory gains in old age. Neuroimage 58: 1110-1120.

47. Heinzel, S., R.C. Lorenz, P. Pelz, et al. 2016. Neural correlates of training and transfer effects in working memory in older adults. Neuroimage 134: 236-249.

48. Qin, Y., C.S. Carter, E.M. Silk, et al. 2004. The change of the brain activation patterns as children learn algebra equation solving. Proc. Natl. Acad. Sci. USA 101: 5686-5691.

49. Haier, R.J., S. Karama, L. Leyba, et al. 2009. MRI assessment of cortical thickness and functional activity changes in adolescent girls following three months of practice on a visual-spatial task. BMC Res. Notes 2: 174.

50. Bialystok, E. 2017. The bilingual adaptation: how minds accommodate experience. Psychol. Bull. 143: 233-262.

51. Lehtonen, M., A. Soveri, A. Laine, et al. 2018. Is bilingualism associated with enhanced executive functioning in adults? A meta-analytic review. Psychol. Bull. 144: 394-425.

52. Abutalebi, J., P.A. Della Rosa, D.W. Green, et al. 2012. Bilingualism tunes the anterior cingulate cortex for conflict monitoring. Cereb. Cortex 22: 2076-2086.

53. Rodríguez-Pujadas, A., A. Sanjuán, P. Fuentes, et al. 2014. Differential neural control in early bilinguals and monolinguals during response inhibition. Brain Lang. 132: 4351.

54. Gold, B.T., C. Kim, N.F. Johnson, et al. 2013. Lifelong bilingualism maintains neural efficiency for cognitive control in aging. J. Neurosci. 33: 387-396.

55. Jolles, D.D. \& E.A. Crone. 2012. Training the developing brain: a neurocognitive perspective. Front. Hum. Neurosci. 6: 76 .
56. Brain Development Cooperative Group. 2012. Total and regional brain volumes in a population-based normative sample from 4 to 18 years: the NIH MRI Study of Normal Brain Development. Cereb. Cortex 22: 1-12.

57. Gogtay, N., J.N. Giedd, L. Lusk, et al. 2004. Dynamic mapping of human cortical development during childhood through early adulthood. Proc. Natl. Acad. Sci. USA 101: 8174-8179.

58. Shaw, P., N.J. Kabani, J.P. Lerch, et al. 2008. Neurodevelopmental trajectories of the human cerebral cortex. J. Neurosci. 28: 3586-3594.

59. Westlye, L.T., K.B. Walhovd, A.M. Dale, et al. 2010. Differentiating maturational and aging-related changes of the cerebral cortex by use of thickness and signal intensity. Neuroimage 52: 172-185.

60. Casey, B.J., R.J. Trainor, J.L. Orendi, et al. 1997. A developmental functional MRI study of prefrontal activation during performance of a go-no-go task. J. Cogn. Neurosci. 9: 835847.

61. Tamm, L., V. Menon \& A.L. Reiss. 2002. Maturation of brain function associated with response inhibition. J. Am. Acad. Child Adolesc. Psychiatry 41: 1231-1238.

62. Velanova, K., M.E. Wheeler \& B. Luna. 2008. Maturational changes in anterior cingulate and frontoparietal recruitment support the development of error processing and inhibitory control. Cereb. Cortex 18: 2505-2522.

63. Booth, J.R., D.D. Burman, J.R. Meyer, et al. 2003. Neural development of selective attention and response inhibition. Neuroimage 20: 737-751.

64. Durston, S., M.C. Davidson, N. Tottenham, et al. 2006. A shift from diffuse to focal cortical activity with development. Dev. Sci. 9: 1-8.

65. Ordaz, S.J., W. Foran, K. Velanova, et al. 2013. Longitudinal growth curves of brain function underlying inhibitory control through adolescence. J. Neurosci. 33: 18109-18124.

66. Simmonds, D.J., M.N. Hallquist \& B. Luna. 2017. Protracted development of executive and mnemonic brain systems underlying working memory in adolescence: a longitudinal fMRI study. Neuroimage 157: 695-704.

67. Wetherill, R.R., L.M. Squeglia, T.T. Yang, et al. 2013. A longitudinal examination of adolescent response inhibition: neural differences before and after the initiation of heavy drinking. Psychopharmacology 230: 663-671.

68. Schulze, K., K. Mueller \& S. Koelsch. 2011. Neural correlates of strategy use during auditory working memory in musicians and non-musicians. Eur. J. Neurosci. 33: 189-196.

69. Schulze, K., S. Zysset, K. Mueller, et al. 2011. Neuroarchitecture of verbal and tonal working memory in nonmusicians and musicians. Hum. Brain Mapp. 32: 771-783.

70. Pallesen, K.J., E. Brattico, C.J. Bailey, et al. 2010. Cognitive control in auditory working memory is enhanced in musicians. PLoS One 5: e11120.

71. Alain, C., Y. Khatamian, Y. He, et al. 2018. Different neural activities support auditory working memory in musicians and bilinguals. Ann. N.Y. Acad. Sci. 1423: 432-443.

72. Sachs, M., J. Kaplan, A. Der Sarkissian, et al. 2017. Increased engagement of the cognitive control network associated with music training in children during an fMRI Stroop task. PLoS One 12: e0187254. 
73. Sala, G. \& F. Gobet. 2017. When the music's over. Does music skill transfer to children's and young adolescents' cognitive and academic skills? A meta-analysis. Educ. Res. Rev. 20: $55-67$.

74. Ullén, F., D.Z. Hambrick \& M.A. Mosing. 2016. Rethinking expertise: a multifactorial gene-environment interaction model of expert performance. Psychol. Bull. 142: 427.
75. Friedman, N.P., A. Miyake, S.E. Young, et al. 2008. Individual differences in executive functions are almost entirely genetic in origin. J. Exp. Psychol. 137: 201225.

76. Schellenberg, E.G. 2015. Music training and speech perception: a gene-environment interaction. Ann. N.Y. Acad. Sci. 1337: $170-177$. 